Psychotherapeut 2020 - 65:93-100 https://doi.org/10.1007/s00278-020-00403-3 Online publiziert: 18. Februar 2020

(c) Der/die Autor(en) 2020
Katrin Schoenenberg • Alexandra Martin

Klinische Psychologie und Psychotherapie, Bergische Universität Wuppertal, Wuppertal, Deutschland

\title{
Bedeutung von Instagram und Fitspiration-Bildern für die muskeldysmorphe Symptomatik
}

\author{
Internalisierung des männlichen \\ Schönheitsideals durch soziale Medien
}

\begin{abstract}
Zahlreiche Studien untersuchen den Einfluss von Medien auf das Körperbild bei Frauen. Der Körper des Mannes stellt jedoch seit einigen Jahren ein ebenso relevantes Thema in den Medien dar. Das mesomorphe Ideal kann oft nur durch ausgeprägten Kraftsport erreicht werden. Neue Medien wie soziale Netzwerke bieten die Möglichkeit des ständigen Vergleichs, weshalb ihre Wirkung für die Forschung von besonderem Interesse ist. Die vorliegende Studie betrachtet aus diesem Grund fokussiert den Zusammenhang zwischen der Nutzung neuer Medien und einem pathologischen Körperbild bei sportlichen Männern.
\end{abstract}

\section{Grundlagen}

Eine negative Einstellung zum eigenen Körper und deutliche Bestrebungen, die Unzufriedenheit zu verändern, werden als Faktoren angesehen, die die Entwicklung von Essstörungen und der körperdysmorphen Störung begünstigen. Das männliche Schönheitsideal orientiert sich nicht an einem möglichst schmalen Körper. Wie Pope et al. (2000) zeigten, schätzen Männer westlich geprägter Länder den idealen Körper eines Mannes im Durchschnitt ca. $13 \mathrm{~kg}$ muskulöser als den eigenen ein. Das Streben nach diesem scheinbaren Ideal, mit einem ausgeprägten V-förmigen Oberkörper und geringem Körperfettanteil (Frederick et al. 2007; Olivardia et al.
2004), wird als das Streben nach Muskulosität bezeichnet (McCreary und Sasse 2000; Waldorf et al. 2014). Es ist assoziiert mit einem niedrigen Selbstwert und höherer Depressivität (Hildebrandt et al. 2004; McCreary, 2007; Thompson und Cafri 2007). Es kann mit restriktivem Essverhalten, das den Muskelaufbau fördert und den Körperfettanteil reduziert, einhergehen, weshalb eine pathologische Ausprägung Ähnlichkeiten zu Essstörungen aufweist (Murray et al. 2012).

Das DSM-5 klassifiziert das unter dem Begriff Muskeldysmorphie bekannte Störungsbild als Subtyp der körperdysmorphen Störung (American Psychiatric Association 2013). Aus diagnostischer Perspektive stellt die ständige Beschäftigung mit dem empfundenen Makel „Schmächtigkeit", der objektiv nicht oder nur in geringem Maß vorliegt, das erste wichtige Kriterium dar. Darüber hinaus beschäftigen sich Betroffene mental oder durch wiederholte Verhaltensweisen mit dem Makel. Im Fall von Muskeldysmorphie äußert sich dies auf der Verhaltensebene beispielsweise durch ausgeprägtes Muskeltraining, häufige Spiegelbetrachtung, die Vermeidung, den Körper in der Öffentlichkeit zu zeigen, und teilweise durch das Einnehmen von anabolen Steroiden.

Seit vielen Jahren wird untersucht, ob Medienkonsum einen negativen Einfluss auf die Entwicklung von pathologischen körperbezogenen Einstellungen bei Frauen hat; deutlich seltener wurde dies bei männlichen Stichproben untersucht. Für Männer werden im Review von Blond (2008) kleine signifikante negative Effekte der Exposition mit Bildern oder Werbung auf die Körperunzufriedenheit beschrieben. Die Metaanalyse von Hausenblas et al. (2013) zeigt kleine Effekte für erhöhte Depressivität, verminderten Selbstwert und erhöhte Wut nach Exposition mit Bildern von idealen Körpern. Depressivität und Körperunzufriedenheit waren in Hochrisikogruppen ausgeprägter. Das Geschlecht stellte keinen Moderator dar, weshalb die Autoren ähnliche Effekte für beide Geschlechter annahmen.

Nach dem „tripartite influence model“ (van den Berg et al. 2002) wirken sich besonders zwei Faktoren auf den $\mathrm{Zu}$ sammenhang von sozialem Einfluss und negativem Körperbild oder Körperunzufriedenheit aus: die Internalisierung des medialen Schönheitsideals und die Tendenz zu aussehensbezogenen Vergleichen. Dabei werden neben den Eltern und Peers die Medien als Quelle des sozialen Einflusses angesehen. Ein negatives Körperbild oder Körperunzufriedenheit führt wiederum zu gestörtem Essverhalten oder muskelaufbauendem Verhalten (Tylka 2011). Die Annahmen des Modells fanden im Kontext der Essstörungen in verschiedenen Studien Bestätigung (Groesz et al. 2002; López-Guimerà et al. 2010; Tiggemann und Miller 2010).

Eine Metaanalyse von Meyrs und Crowther (2009) zeigte positive $\mathrm{Zu}$ sammenhänge zwischen Tendenzen zu sozialem Vergleich und Körperunzufriedenheit, jedoch waren diese stärker bei Frauen als bei Männern ausgeprägt. In 
einer Studie von Klimek et al. (2018) stellte sowohl die Internalisierung des muskulösen als auch des schlanken medialen Schönheitsideals einen Prädiktor für muskeldysmorphe Symptomatik und gestörtes Essverhalten bei Männern dar.

Mit der Verbreitung und Entwicklung sozialer Medien ist auch deren Einfluss in das Interesse der Forschung gerückt. Hinsichtlich der Auswirkungen auf das Körperbild bringt diese Form der Medien besondere Risiken mit sich. Der sozialen Vergleichstheorie zufolge bevorzugen Menschen Vergleiche mit Personen, die ihnen ähnlich sind (Festinger 1954). Soziale Netzwerke bieten die perfekte Möglichkeit des Vergleichs und sind darüber hinaus ständig verfügbar.

Ein aktuelles Review von Holland und Tiggemann (2016) berichtet einen positiven Zusammenhang zwischen der Zeit oder der Häufigkeit der Nutzung sozialer Netzwerke und dem Körperbild. In 5 von 6 Studien konnte kein Geschlechterunterschied gefunden werden. In den analysierten Studien wurde hauptsächlich das Netzwerk Facebook untersucht. Die Nutzung von Facebook oder Instagram scheint jedoch zumindest bei Frauen nur dann mit der Internalisierung des schlanken Schönheitsideals oder der Körperunzufriedenheit zusammenzuhängen, wenn aussehensbezogene Inhalte konsumiert werden (Brown und Tiggemann 2016; Cohen et al. 2017; Tiggemann und Miller 2010). Fardouly et al. (2018) untersuchten bei Frauen, ob die generelle Nutzung von Instagram oder Nutzung von „Fitspiration“-Bildstrecken mit Körperunzufriedenheit und dem Sterben nach Schlankheit assoziiert war. Fitspiration-Bilder dienen der Motivationssteigerung („inspiration“), einen möglichst "fitten“ Körper zu erhalten. Die dort gezeigten Bilder vermitteln den Eindruck, dass jeder das Schönheitsideal durch ausreichende Bemühungen erreichen kann. In den Ergebnissen bestand ein signifikanter Zusammenhang zwischen den untersuchten Konstrukten und dem Betrachten von FitspirationBildern, nicht aber mit der allgemeinen Nutzungshäufigkeit von Instagram. Die Internalisierung des medialen Schönheitsideals und der Tendenz zu ausse- hensbezogenen Vergleichen mediierten die Zusammenhänge.

\section{Ziel der Arbeit}

Die dargestellten Befunde zur Nutzung sozialer Medien und dem Körperbild wurden überwiegend in Studien mit weiblichen Personen gewonnen. Es stellt sich die Frage, ob die Ergebnisse auch auf männliche Stichproben übertragen werden können. In diesem Kontext ist ebenfalls offen, ob die Internalisierung des Schönheitsideals und aussehensbezogene Vergleiche von ähnlicher Relevanz für Männer sind. Darüber hinaus möchte die Studie klären, ob Kraftsportler eine Hochrisikogruppe für die Entwicklung von pathologischen Einstellungen und pathologischem Verhalten darstellen. Besonders kraftsportausübende Männer könnte das Betrachten anderer Sportler auf Instagram motivieren, den muskulösen Idealkörper zu erreichen. Es ergibt sich die Frage, ob sich Kraftsportler von Personen, die auch andere Sportarten ausüben, unterscheiden.

\section{Methodik}

\section{Studiendesign und Stichprobe}

Männer im Alter von 18 bis 65 Jahren, die Sport ausübten und Instagram nutzten, wurden im Rahmen einer Querschnittserhebung über eine Onlinebefragung untersucht.

Die Stichprobe umfasste junge Männer $(n=203)$ im mittleren Alter von 27,98 Jahren (SD $\pm 7,50$ Jahre). Etwas über die Hälfte der Personen berichtete, in einer festen Beziehung oder verheiratet zu sein (55,7\%). Das Abitur oder einen höheren Schlussabschluss hatten $71,9 \%$, die Fachhochschulreife $12,8 \%$, den Real- oder Hauptschulabschluss 15,2\% der Teilnehmer. Der mittlere Body-Mass-Index (BMI) be$\operatorname{trug} 25,63 \mathrm{~kg} / \mathrm{m}^{2}\left(\mathrm{SD} \pm 4,77 \mathrm{~kg} / \mathrm{m}^{2}\right)$. Alle Teilnehmer nutzten Instagram; ein Teil nutzte darüber hinaus andere soziale Netzwerke, wie Facebook ( $n=195)$ oder YouTube $(n=153)$.

Die meisten Teilnehmer gaben an, Kraftsport (85,7\%) oder einen Ausdauersport $(56,2 \%)$ zu betreiben. Ein Teil war in Ballsportarten aktiv (33,0\%) oder in anderen Sportarten wie Kampfsport (28,1\%). Hinsichtlich der Anzahl der Sportarten waren $26,6 \%$ in den letzten 4 Wochen in einer, $42,9 \%$ in $2,23,6 \%$ in 3 und $4 \%$ in 4 oder 5 Sportarten aktiv.

\section{Untersuchungsablauf}

Die Teilnehmer wurden in sozialen Netzwerken, über Flyer in Fitnessstudios und an der Bergischen Universität Wuppertal rekrutiert. Voraussetzungen zur Teilnahme waren männliches Geschlecht, regelmäßiges Ausüben von Sport und die Nutzung von Instagram. Anhand eines Links konnte auf den Onlinefragebogen zugegriffen werden. Nach der Information über die Ziele und den Zweck der Studie sowie den Umgang mit den erhobenen Daten und dem Datenschutz wurde in die Teilnahme eingewilligt. Die Bearbeitung des Fragbogens dauerte im Mittel 19,45 min (SD $\pm 4,83 \mathrm{~min}$ ).

\section{Instrumente}

Demografische Fragen. Die Teilnehmer wurden gebeten, Alter, Geschlecht, Beziehungsstatus, höchsten Schulabschluss, Körpergewicht und -größe anzugeben.

Sportliche Aktivität. Aus 5 Sportkategorien wählten die Teilnehmer alle Sportarten aus, die sie innerhalb der vergangenen 4 Wochen betrieben hatten (Kraftsport, Ausdauersport, Ballsportarten, andere körperintensive Sportarten und Sonstige; in Anlehnung an Weineck 2010).

Instagram-Nutzung. In Anlehnung an Junco et al. (2011) wurde die Zeit, die pro Tag mit 12 verschiedenen Aktivitäten auf Instagram verbracht wurde (z. B. Status-Update checken, kommentieren, Fotos hochladen u. Ä.), mithilfe der LikertSkalen von 1: „nie“ bis 5: „sehr häufig“ erfasst. Der Summenwert der 12 Items bildete den Wert für die generelle Nutzungshäufigkeit von Instagram (NI). Für eine gute Reliabilität spricht die resultierende interne Konsistenz (Cronbachs $\alpha=0,83$ ). Die spezifische Nutzung von Fitspiration-Bildern $\left(\mathrm{NI}_{\mathrm{F}}\right)$ wurde mit dem Item „Wie häufig sehen Sie sich \#Fitspiration- 
Psychotherapeut 2020 - 65:93-100 https://doi.org/10.1007/s00278-020-00403-3

(c) Der/die Autor(en) 2020

\section{K. Schoenenberg · A. Martin}

\section{Bedeutung von Instagram und Fitspiration-Bildern für die muskeldysmorphe Symptomatik. Internalisierung des männlichen Schönheitsideals durch soziale Medien}

\section{Zusammenfassung}

Hintergrund. Das männliche Körperideal unterscheidet sich von dem weiblichen durch eine Orientierung am muskulösen Körperbau. Diese Studie untersucht, inwiefern der Konsum von Instagram das Streben nach Muskulosität und muskeldysmorpher Symptomatik bei Männern vorhersagt. Darüber hinaus wird geprüft, ob relevante Mediatoren die Zusammenhänge in einer männlichen Stichprobe beeinflussen. Methodik. Es wurden sportliche, Instagram nutzende Männer befragt $(n=203)$. Neben der Nutzungshäufigkeit von Instagram im Allgemeinen und der Betrachtung von Fitspiration-Bildern im Speziellen wurden das Streben nach Muskulosität, die Ausprägung muskeldysmorpher Symptomatik, die Tendenz zu aussehensbezogenen Vergleichen und die Internalisierung des medialen Schönheitsideals erfasst.

Ergebnisse. Gruppenvergleiche wiesen auf Unterschiede zwischen Männern, die zusätzlich Ballsport ausübten, zu solchen, die hauptsächlich Kraftsport trieben, hin. Die Internalisierung des medialen Schönheitsideals mediierte den Zusammenhang zwischen der allgemeinen und spezifischen Nutzungshäufigkeit von Instagram und dem Streben nach Muskulosität sowie der Ausprägung muskeldysmorpher Symptomatik. Diskussion. Die Ergebnisse zeigen den Zusammenhang zwischen der Nutzungshäufigkeit von Instagram und Facetten des männlichen Körperbilds. Sie verdeutlichen ebenfalls die Bedeutung der Internalisierung des medialen Schönheitsideals bei der Nutzung sozialer Medien. Kraftsport ausübende Männer wiesen im Selbstbericht ein höheres Risiko für eine muskeldysmorphe Symptomatik auf. Die Nutzung sozialer Medien könnte im Rahmen einer muskeldysmorphen Störung eine aufrechterhaltende Funktion einnehmen.

Schlüsselwörter Körperdysmorphe Störung · Essstörungen Körperbild· Soziale Medien · Sport

\section{Relevance of Instagram and Fitspiration images for muscle dysmorphia. Internalization of the male beauty ideal through social media}

Abstract

Background. The male body ideal differs from the female ideal because males strive for a strong and muscular physique. This study investigated how far the use of Instagram predicts the drive for muscularity and muscle dysmorphia in men. The second aim was to test whether relevant mediators influence the relationships in a random sample of men. Material and methods. Exercising men $(n=203)$ who were using Instagram participated in the questionnaire-based survey. Besides the extent of general Instagram use and specific use of Fitspiration images, the drive for muscularity, extent of muscle dysmorphia symptoms, the internalization of the media beauty ideal and the tendency towards appearance-based comparisons were assessed.

Results. Group comparisons indicated differences between men who were additionally doing ball sports to those who were only doing body-building. The internalization of the media beauty ideal mediated the relationship between general and specific Instagram use and the drive for muscularity as well as the extent of symptoms of muscle dysmorphia.
Conclusion. The results showed a relationship between Instagram use and facets of the male body image. They further emphasized the importance of the internalization of the media beauty ideal when using social media. Men practicing body-building showed a higher self-reported risk for symptoms of muscle dysmorphia. Using social media could constitute a maintaining function in a muscle dysmorphic disorder.

Keywords

Body dysmorphic disorder - Eating disorders . Body image $\cdot$ Social media $\cdot$ Sports
Bilder bei einem Besuch auf Instagram an?" erhoben (Fardouly et al. 2018). Das Item mit einer Antwortskala von 1: „nie“ bis 5: „immer" wurde separat erfasst und ging nicht in die NI-Summenwert-Berechnung ein.

Streben nach Muskulosität. Die Drive for Muscularity Scale (DMS) erfasst das Muskulositätsstreben in 15 Items, die auf einer Likert-Skala von 1: „immer" bis 6: „nie“ beantwortet werden; Beispielitem: „Ich wünschte, ich wäre muskulöser“. Sowohl das englische Original (McCreary und Sasse 2000) als auch die deutsche
Übersetzung (Waldorf et al. 2014) sind reliable und valide Instrumente zur Erfassung des Konstrukts; in der vorliegenden Stichprobe betrug die interne Konsistenz $a=0,91$. Für den Grad des Muskulositätsstrebens wurde der Gesamtmittelwert verwendet.

Muskeldysmorphe Symptomatik. Das Muscle Dysmorphic Disorder Inventory (MDDI) von Hildebrandt et al. (2004) erfasst in 13 Items die Ausprägung von Symptomen der muskeldysmorphen Störung. Da zum Zeitpunkt der Erhebung keine validierte deutsche Version vorlag, wurden die Items zunächst ins Deutsche und zur Prüfung erneut ins Englische übersetzt. Die Items wurden auf einer Likert-Skala von 1: „nie“ bis 5: „immer“ beantwortet, Beispielitems sind „Ich finde meinen Körper zu schmächtig.. „Ich hasse meinen Körper“. In der vorliegenden Stichprobe resultierte eine gute interne Konsistenz von $\alpha=0,84$.

\section{Selbstscreening zur körperdysmor-} phen Störung. Das Vorliegen einer körperdysmorphen Störung wurde über 8 Items erhoben, die die DSM-5-Kriterien und die Intensität der Beschäftigung 
Tab. 1 Deskriptive Ergebnisse und Korrelationen der Variablen

\begin{tabular}{|c|c|c|c|c|c|c|c|c|}
\hline & \multirow[t]{2}{*}{$M$} & \multirow[t]{2}{*}{ SD } & \multirow{2}{*}{$\begin{array}{l}\text { Minimum bis } \\
\text { Maximum }\end{array}$} & \multicolumn{5}{|c|}{ Korrelationen } \\
\hline & & & & 2 & 3 & 4 & 5 & 6 \\
\hline 1. NI & 33,05 & $\pm 8,83$ & $12-59$ & $0,33^{* *}$ & $0,44^{* *}$ & $0,46^{* *}$ & $0,45^{* *}$ & $0,82^{* *}$ \\
\hline 2. $\mathrm{NI}_{\mathrm{F}}$ & 2,25 & $\pm 1,28$ & $1-5$ & - & $0,38^{* *}$ & $0,37^{* *}$ & $0,18^{*}$ & $0,37^{*}$ \\
\hline 3. DMS & 3,25 & $\pm 1,10$ & $1,13-5,80$ & - & - & $0,70 * *$ & $0,30 * *$ & $0,62^{* *}$ \\
\hline 4. MDDI & 31,72 & $\pm 8,95$ & $15-58$ & - & - & - & $0,26^{* *}$ & $0,53^{* *}$ \\
\hline 5. PACS & 8,16 & $\pm 3,78$ & $0-20$ & - & - & - & - & $0,42^{* *}$ \\
\hline 6. SATAQ-G.int & 16,52 & $\pm 5,57$ & $6-30$ & - & - & - & - & - \\
\hline
\end{tabular}

$n=203,{ }^{* *} p<0,01,{ }^{*} p<0,05$

DMS Drive for Muscularity Scale, M Mittelwert, MDDI muskeldysmorphe Symptomatik, NI Nutzungshäufigkeit von Instagram, $N I_{F}$ Nutzungshäufigkeit von Fitspiration-Bildern, $P A C S$ aussehensbezogene Vergleiche, SATAQ-G.int Internalisierung des medialen Schönheitsideals, SD Standardabweichung

Tab. 2 Deskriptive Ergebnisse für Subgruppen

\begin{tabular}{lll|l|l|l|l} 
& \multicolumn{2}{l}{$\begin{array}{l}\text { Kraftsport } \\
\boldsymbol{n}=\mathbf{4 7}\end{array}$} & & \multicolumn{2}{l}{$\begin{array}{l}\text { Kraft- und Ausdauersport } \\
\boldsymbol{n}=\mathbf{6 7}\end{array}$} & $\begin{array}{l}\text { Kraft- und Ballsport } \\
\boldsymbol{n}=\mathbf{4 9}\end{array}$ \\
& $\mathbf{M}$ & SD & $\mathbf{M}$ & SD & $\mathbf{M}$ & SD \\
\hline $\mathrm{NI}$ & 35,06 & $\pm 9,06$ & 33,70 & $\pm 9,10$ & 32,53 & $\pm 8,24$ \\
\hline $\mathrm{NI}_{F}$ & 2,68 & $\pm 1,35$ & 2,45 & $\pm 1,18$ & 1,96 & $\pm 1,29$ \\
\hline DMS & 3,98 & $\pm 0,99$ & 3,35 & $\pm 1,03$ & 3,01 & $\pm 0,94$ \\
\hline MDDI & 35,85 & $\pm 9,44$ & 32,57 & $\pm 9,45$ & 29,14 & $\pm 7,33$ \\
\hline PACS & 8,15 & $\pm 4,01$ & 7,97 & $\pm 3,50$ & 9,02 & $\pm 3,67$ \\
\hline SATAQ-G.int & 18,47 & $\pm 5,76$ & 16,81 & $\pm 5,60$ & 16,02 & $\pm 5,01$
\end{tabular}

Kategorie Kraft- und/oder Sonstiger Sport: ausschließlich sonstiger Sport $n=29$, Kraft- und sonstiger Sport $n=11$. Aufgrund von Heterogenität nicht aufgeführt

$D M S$ Drive for Muscularity Scale, $M$ Mittelwert, $M D D I$ muskeldysmorphe Symptomatik, $N I_{F}$ Nutzungshäufigkeit von Fitspiration-Bildern, PACS aussehensbezogene Vergleiche, SATAQ-G.int Internalisierung des medialen Schönheitsideals, SD Standardabweichungen

mit aussehensbezogenen Gedanken und Verhalten abfragen (Schieber et al. 2015). Das letzte Item stellt eine kurze Makelliste der häufigsten Makelbereiche dar.

Aussehensbezogene Vergleiche. Die Physical Appearance Comparison Scale (PACS; dt. Version von Mölbert et al. 2017) erfasst in 5 Items die Tendenz, sich mit anderen Personen bezüglich des Aussehens zu vergleichen. Antworten wurden auf einer Likert-Skala von 0: „nie“ bis 4: „immer“ gegeben. In der vorliegenden Studie resultierte eine etwas höhere interne Konsistenz $(\alpha=0,67)$ als in der Validierungsstudie für die männliche Stichprobe $(\alpha=0,59)$.

\section{Internalisierung des medialen Schön-} heitsideals. Die deutsche Version des Sociocultural Attitudes Towards Appearance Questionnaire (SATAQ-G; Knauss et al. 2009) misst den soziokulturellen Einfluss auf das Körperbild. Die Instruk- tion wurde an die Nutzung von Instagram und das muskulöse Ideal angepasst. Im Rahmen dieser Studie war die mit 6 Items erhobene Subskala Internalisierung (SATAQ-G.int) von besonderem Interesse; ein Beispielitem ist „Mein Ziel ist es, so auszusehen, wie Männer, die man bei Instagram sieht“. Die interne Konsistenz der Subskala betrug $a=0,83$.

\section{Statistische Analyse}

Die Auswertung erfolgte mithilfe des Statistical Package for the Social Sciences (SPSS 25). Zur deskriptiven Analyse der Daten wurden Subgruppen nach der ausgeübten Sportart gebildet (ausschließlich Kraftsport, Kraft- und Ausdauersport, Kraft- und Ballsport) und mithilfe von $t$-Tests verglichen. Levene-Tests dienten vorab der Testung der Varianzhomogenität, die in allen Fällen gegeben war. Die Normalverteilung der Werte innerhalb der Gruppen wurde mithilfe des Kolomogrow-Smirnow-Tests überprüft. Diese war nicht für alle Fälle gegeben. Da verschiedene Studien die Robustheit gegen die Verletzung der Normalverteilungsvoraussetzung zeigen (Bortz und Schuster 2010), wurden die $t$-Tests durchgeführt.

Zur Bewertung von Zusammenhängen der erhobenen Maße in der Gesamtstichprobe wurden Pearson-Korrelationen ermittelt. Die Analyse der Mediationsmodelle erfolgte mithilfe von PROCESS 3.2 für SPSS (Hayes 2019). Dabei wurde die Zahl der Bootstraps auf 10.000 festgelegt. Histogramme der standardisierten Residuen dienten der Überprüfung der Normalverteilungsannahme. Diese war für alle Modelle gegeben. Die Heteroskedastizität der Daten wurde mithilfe des Breusch-Pagan-Tests ermittelt. Da die Heteroskedastizität der Residuen in 2 Modellen verletzt war, wurde für den Signifikanztest und die Berechnung des Konfidenzintervalls ein robuster Standardfehler vom DavidsonMacKinnon-Typ verwendet.

\section{Ergebnisse}

\section{Deskriptive Ergebnisse}

Von den 203 befragten Männern erfüllten 11 (5,4\%) laut Selbstauskunft die Kriterien einer körperdysmorphen Störung (genannte Makelbereiche: 4-mal Haare, einmal Ohren, 2-mal Nase, 2-mal Brust, 3-mal Bauch, einmal Größe und Form der Muskulatur, einmal Po, einmal Hände, 3-mal Haut, einmal Genitalien, einmal Fuß). In - Tab. 1 sind die mittleren Ausprägungen und bivariaten Korrelationen der Variablen aufgeführt. In der Gesamtstichprobe zeigt sich mit ansteigendem Grad des Strebens nach Muskulosität eine stärkere Ausprägung der muskeldysmorphen Symptomatik (•Tab. 1). Die Instagram-Nutzungshäufigkeit weist signifikante moderate Zusammenhänge mit dem Muskulositätsstreben und der muskeldysmorphen Symptomatik auf. Auffallend hoch ist ihr Zusammenhang mit der Internalisierung des medialen Schönheitsideals. Signifikant positive Korrelationen bestehen auch zwischen der Nutzung von Fitspiration und den interessierenden Konstrukten. 


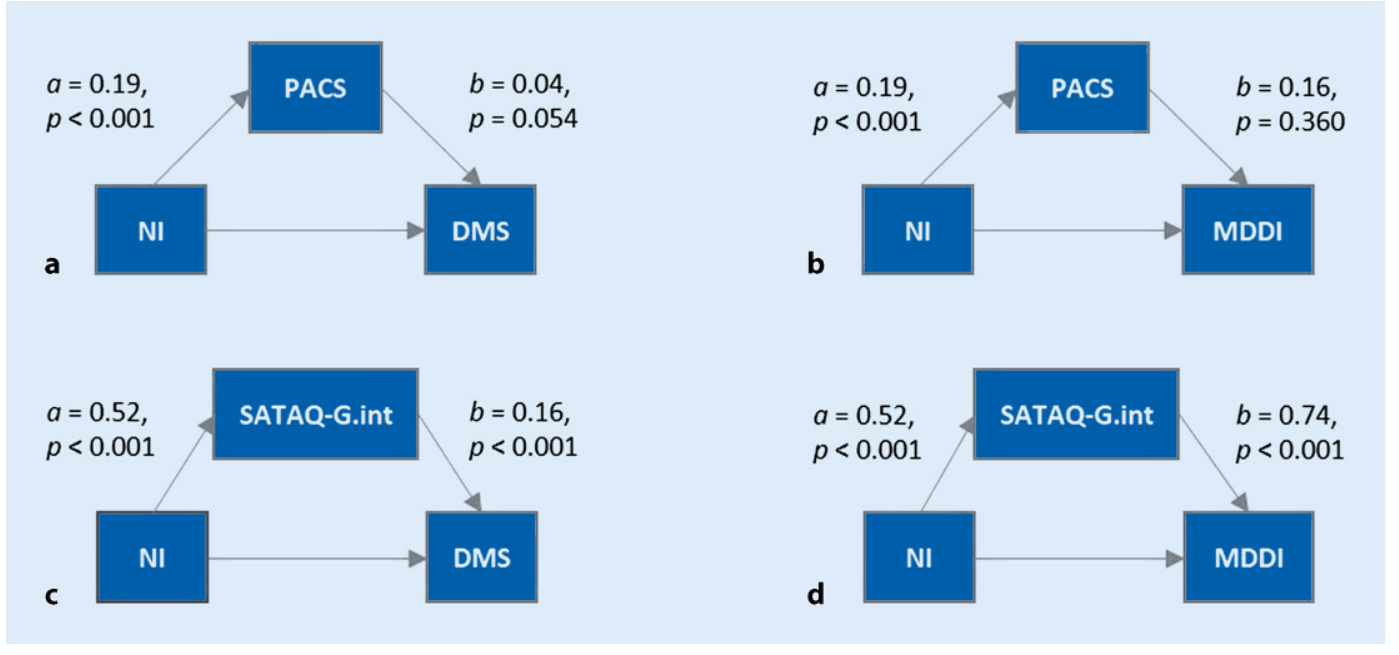

Abb. 1 ॥ Mediationsmodelle für die Mediation von aussehensbezogenen Vergleichen (PACS) und der Internalisierung des medialen Schönheitsideals (SATAQ-G.int) im Zusammenhang mit der Nutzungshäufigkeit von Instagram (NI) mit dem Streben nach Muskulosität (DMS) und mit der Ausprägung von muskeldysmorpher Symptomatik $(M D D I)$. a Totaler Effekt $c=0,05$, $p<0,001$; direkter Effekt $c^{\prime}=0,05 . p<0,001$; indirekter Effekt $\mathrm{a} \cdot \mathrm{b}=0,01 ; 95 \%-\mathrm{KI}[-0,01,0,02] ; \mathrm{Z}=1,85, p=0,064 ; \mathrm{b}$ totaler Effekt $c=0,47, p=0,001$; direkter Effekt $c^{\prime}=0,44, p<0,001$; indirekter Effekt a $\cdot \mathrm{b}=0,03,95 \%$-KI $[-0,03,0,10], \mathrm{Z}=0,90, p=0,367$; ctotalerEffekt $\mathrm{c}=0,05, p<0,001$; direkterEffekt $\mathrm{c}^{\prime}=-0,03, p=0,018$; indirekterEffekta $\cdot \mathrm{b}=0,08,95 \%-\mathrm{KI}[0,07,0,10], \mathrm{Z}=7,74$, $p<0,001$; d totaler Effekt $c=0,47, p=0,001$; direkter Effekt $c^{\prime}=0,09, p=0,418$, indirekter Effekt $a \cdot b=0,38,95 \%-K I[0,17,0,59]$, $\mathrm{Z}=4,23, p<0,001$

Im Subgruppenvergleich (•Tab. 2) zeigte sich bei ausschließlichen Kraftsportlern ein signifikant höheres Streben nach Muskulosität, $t(112)=3,27, p<0,01$, $d=0,62$, als bei Kraft- und Ausdauersportlern. Im Vergleich von Kraftsportlern zu Kraft- und Ballsportlern weisen Erstere signifikant höhere Werte bezüglich der Nutzungshäufigkeit von Fitspiration-Bildern, $t(94)=2,68, p<0,01$, $d=0,55$, des Strebens nach Muskulosität, $t(94)=4,95, p<0,01, d=1,01$, der Ausprägung der muskeldysmorphen Symptomatik, $t(94)=3,90, p<0,01, d=0,79$, und der Internalisierung des medialen Schönheitsideals auf, $t(94)=2,22$, $p<0,05, d=0,45$.

\section{Mediationsmodelle}

\section{Allgemeine Nutzungshäufigkeit von Instagram und Muskulositäts- streben/muskeldysmorphe Symptomatik}

DasStreben nach Muskulosität $\left(R^{2}=0,19\right.$, $p<0,001)$ und die Ausprägung der muskeldysmorphen Symptomatik $\left(R^{2}=0,21\right.$, $p<0,001)$ können signifikant durch die Nutzungshäufigkeit von Instagram vorhergesagt werden (totaler Effekt, Pfad c; - Abb. 1). Die Nutzungshäufigkeit stellt ebenfalls einen signifikanten Prädiktor für die Tendenz zu aussehensbezogenen Vergleichen $\left(R^{2}=0,20, p<0,001\right)$ und die Internalisierung des medialen Schönheitsideals dar $\left(R^{2}=0,68, p<0,001\right.$; jeweils Pfad a).

Die Tendenz zu aussehensbezogenen Vergleichen erweist sich in beiden Modellen nicht als Mediator (• Abb. 1a, b). Der Sobel-Test zur Prüfung des indirektenEffekts wird nicht signifikant; entsprechend umschließt das Konfidenzintervall die Null.

Die Internalisierung des medialen Schönheitsideals zeigt sich hingegen als signifikanter Mediator in beiden Modellen (• Abb. 1c, d). Im Zusammenhang von Nutzungshäufigkeit und Streben nach Muskulosität kann eine partielle Mediation gefunden werden, da der direkte Effekt (Pfad c') weiterhin signifikant bleibt. Der Zusammenhang von Nutzungshäufigkeit von Instagram und Ausprägung der muskeldysmorphen Symptomatik wird vollständig durch die Internalisierung des medialen Schönheitsideals mediiert.

\section{Nutzungshäufigkeit von Fitspiration-Bildern und Muskulositätsstreben/ muskeldysmorphe Symptomatik}

Die Nutzungshäufigkeit von FitspirationBildern sagt das Streben nach Muskulosität $\left(R^{2}=0,14, p<0,001\right)$ und die Ausprägung der muskeldysmorphen Symptomatik $\left(R^{2}=0,13, p<0,001\right)$ signifikant vorher (totaler Effekt, Pfad c; - Abb. 2). Die Tendenz zu aussehensbezogenen Vergleichen $\left(R^{2}=0,03, p<0,001\right)$ und die Internalisierung des medialen Schönheitsideals $\left(R^{2}=0,13, p<0,001\right.$; jeweils Pfad a) können ebenfalls durch die Nutzungshäufigkeit von Fitspiration-Bildern signifikant vorhergesagt werden. In allen 4 Regressionen ist die Vorhersagekraft geringer als für den Prädiktor allgemeine Nutzungshäufigkeit.

Eine partielle Mediation durch die Tendenz zu aussehensbezogenen Vergleichen wurde im Fall von Streben nach Muskulosität gefunden (• Abb. 2a). In der Vorhersage der muskeldysmorphen Symptomatik ist die Null zwar nicht im Konfidenzintervall enthalten, der SobelTest wird jedoch knapp nicht signifikant (• Abb. 2b).

Bezüglich des Mediators Internalisierung des medialen Schönheitsideals las- 


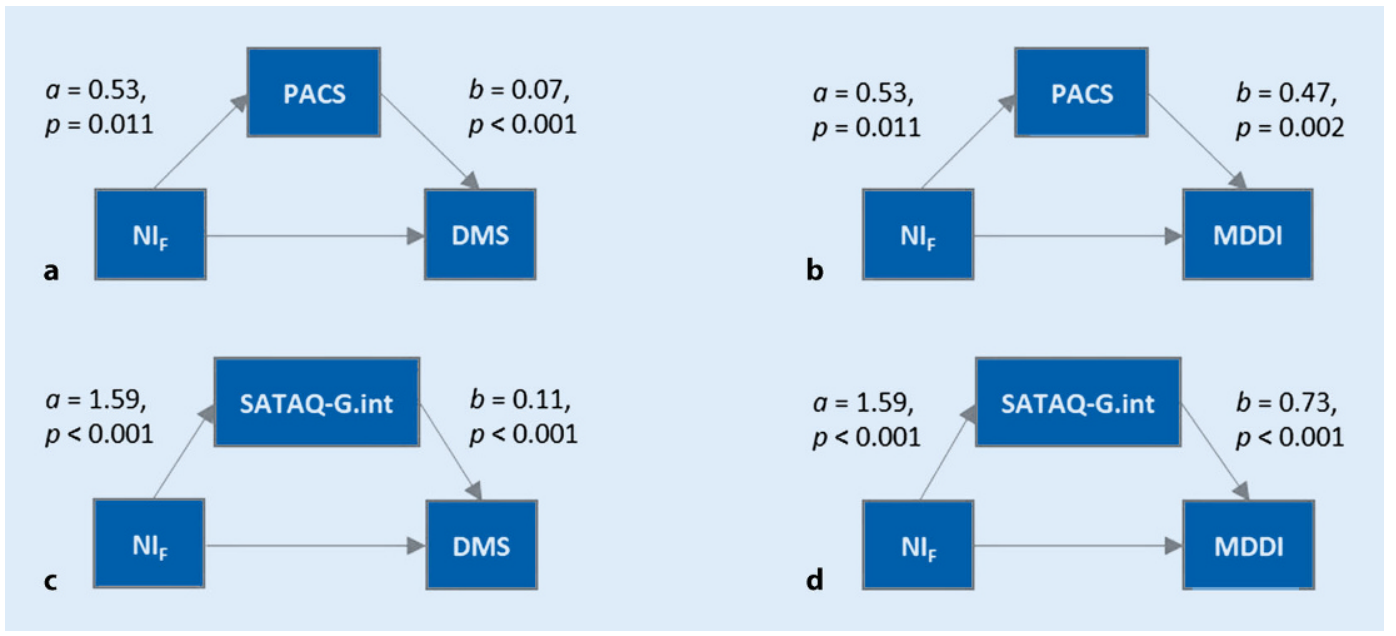

Abb. 2 A Mediationsmodelle für die Mediation von aussehensbezogenen Vergleichen (PACS) und der Internalisierung des medialen Schönheitsideals (SATAQ-G.int) im Zusammenhangmit der Nutzungshäufigkeit von Fitspiration-Bildern auf Instagram $\left(N I_{F}\right)$ mit dem Streben nach Muskulosität $(D M S)$ und mit der Ausprägung von muskeldysmorpher Symptomatik $(M D D I)$. a Totaler Effekt $c=0,33, p<0,001$; direkter Effekt $c^{\prime}=0,29, p<0,001$; indirekter Effekt $\mathrm{a} \cdot \mathrm{b}=0,04 ; 95 \%$-KI $[0,01-0,08]$; $\mathrm{Z}=2,08, p=0,038$; $\mathbf{b}$ totaler Effekt $\mathrm{c}=2,56, p=0,001$; direkter Effekt $\mathrm{c}^{\prime}=2,31, p<0,001$; indirekter Effekt $\mathrm{a} \cdot \mathrm{b}=0,25,95 \%-\mathrm{KI}$ $[0,04-0,56], \mathrm{Z}=1,92, p=0,055$; c totaler Effekt $\mathrm{c}=0,33, p<0,001$; direkter Effekt $\mathrm{c}^{\prime}=0,15, p=0,003$; indirekter Effekt $\mathrm{a} \cdot \mathrm{b}=0,17,95 \%$-KI $[0,11,0,25], Z=4,79, p<0,001$; d totaler Effekt $c=2,56, p=0,001$; direkter Effekt $c^{\prime}=1,40, p=0,002$, indirekter Effekt $\mathrm{a} \cdot \mathrm{b}=1,17,95 \%-\mathrm{KI}[0,66,1,77], \mathrm{Z}=4,19, p<0,001$

sen sich für beide Kriterien partielle Mediationen finden (• Abb. 2c, d).

\section{Diskussion}

\section{Interpretation der Ergebnisse}

Das Ziel der Studie war, den Zusammenhang zwischen der Nutzung von Instagram und Variablen des Körperbilds bei Männern zu überprüfen. Darüber hinaus sollten Mediatoren, die mit diesem $\mathrm{Zu}$ sammenhang in der Literatur bei weiblichen Stichproben gefunden wurden, für eine männliche Stichprobe geprüft werden.

Im Subgruppenvergleich unterschieden sich die ausschließlich kraftsportausübenden Männer hinsichtlich eines stärker ausgeprägten Strebens nach Muskulosität von Kraft- und Ausdauersportlern. Die Kraftsportler betrachteten darüber hinaus deutlich häufiger Fitspiration-Bilder, strebten stärker nach Muskulosität und berichteten eine ausgeprägtere muskeldysmorphe Symptomatik als Kraft- und Ballsportler. Da keine Priorität der Sportarten erhoben wurde, können deren Rollen für die Beurteilung der Befunde nicht mitberücksichtigt werden. Eine Erklärung könnte jedoch darin liegen, dass Ballsportler Kraftsport eher als
Ergänzung ausüben und nicht, um primär optisch einen bestimmten Körperbau zu erlangen. Diese Hypothese sollte in nachfolgenden Studien näher geprüft werden.

Auf korrelativer Ebene zeigten sich entsprechend den Annahmen positive Zusammenhänge zwischen dem Streben nach Muskulosität und dem Ausmaß der muskeldysmorphen Symptomatik mit der Nutzungshäufigkeit von Instagram (generell und spezifisch). Darüber hinaus bestanden hohe Korrelationen zu den untersuchten Mediatoren. Die spezifische Nutzung von Fitspiration-Bildern weist im Vergleich zur generellen Nutzung geringe Zusammenhänge mit der Internalisierung des Medienideals und der Tendenz zu aussehensbezogenen Vergleichen auf. Dies könnte mit einer geringeren Messgenauigkeit des einzelnen Items in Verbindung stehen.

In den geprüften Mediationsmodellen stellte die Internalisierung des medialen Schönheitsideals in allen getesteten Modellen einen Mediator dar. Im Fall von allgemeiner Nutzungshäufigkeit und muskeldysmorpher Symptomatik handelte es sich um eine volle Mediation. Inwieweit die teilnehmenden Männer dem gängigen Schönheitsideal Zuspruch entgegenbrachten, erklärte somit teilweise bzw. vollständig die Ausprägung des Strebens nach Muskulosität und der muskeldysmorphen Symptomatik. Damit stützen die Ergebnisse die Annahmen des tripartite influence model und dessen Erweiterung (van den Berg et al. 2002; Tylka 2011).

Die Tendenz zu aussehensbezogenen Vergleichen erwies sich nur im Zusammenhang mit der Nutzung von Fitspiration-Bildern als partieller Mediator. Die Ergebnisse stehen im Einklang mit den Befunden von Karazsia und Crowther (2009), die in der Vorhersage des Strebens nach Muskulosität durch sozialen Einfluss sowohl die Internalisierung als auch aussehensbezogene Vergleiche als mediierende Faktoren zeigten. Darüber hinaus bestätigen sie die spezifische Wirkung, die von Fitspiration-Bildern ausgehen könnte (u.a. Brown und Tiggemann 2016; Fardouly et al. 2018).

Für den Behandlungskontext stellt sich die Frage, ob die diskutierten Mediatorvariablen veränderbar sind. Eine der wenigen Behandlungsstudien mit körperunzufriedenen Männern konnte eine Reduktion der Internalisierung des Schönheitsideals in einer Interventionsgruppe im Vergleich zu einer Wartegruppe finden, die auch zum VierwochenFollow-up stabil war (Brown et al. 2017). 
Dies bietet erste Hinweise auf mögliche Ansatzpunkte im therapeutischen Kontext.

\section{Limitationen und Ausblick}

Diese Studie stellt insgesamt eine der wenigen dar, die den Einfluss von neuen Medien auf das männliche Körperbild untersucht. Bewusst wurde eine Stichprobe von hauptsächlich kraftsportreibenden Männern rekrutiert. Die Erwartungen von hohen Zusammenhängen zwischen der Nutzung sozialer Medien und dem gezielten Muskelaufbau zur Erreichung eines bestimmten Schönheitsideals konnten für diese Gruppe bestätigt werden. Es kann vermutet werden, dass die Effekte für Männer, die fokussiert eine andere Sportart oder keinen Sport ausüben, geringer ausfallen würden. Die Ergebnisse sind vergleichbar mit den Befunden bei Frauen (Fardouly et al. 2018), in denen hingegen ein Zusammenhang zwischen allgemeiner Instagram-Nutzung und Körperunzufriedenheit nicht gezeigt werden konnte.

Die untersuchte Stichprobe wies unterschiedlich stark ausgeprägte muskeldysmorphe Symptome auf, und ein positives Screening für eine körperdysmorphe Störung lag bei 5,4\% der Männer vor. Die Prävalenz ist verglichen mit Angaben, die die deutsche Allgemeinbevölkerung betreffen, erhöht (Schieber et al. 2015). Inwieweit sich die Zusammenhänge allerdings in einer Stichprobe von Personen mit einer Muskeldysmorphiediagnose abbilden, bleibt ungeklärt. Besonders für die Einordnung der Rolle sozialer Medien in der Therapie ist es wichtig, die hier geprüften Hypothesen auch für rein klinische Stichproben zu bestätigen.

Als weitere Limitationen sind das Querschnittsdesign und die Erhebung, basierend auf dem Selbstbericht, zu nennen. Möglicherweise unterscheiden sich die tatsächliche und die wahrgenommene Nutzung, woraus sich die Frage ableitet, welche eher im Zusammenhang mit einem negativen Körperbild steht. Von großem Interesse für die zukünftige Forschung ist, andere Ansätze zur Erfassung internalisierter Körperbilder zu entwickeln.
Die vorliegende Studie fokussiert auf Instagram, da dem sozialen Netzwerk aufgrund der Fotobasierung und Nutzungsstruktur eine besondere Bedeutung für die Ausbildung und Aufrechterhaltung von Schönheitsidealen zugesprochen werden könnte. Diese Wirkung sollte in zukünftiger Forschung genauer untersucht werden. Besonders im Kontext der körperdysmorphen Störung hat der Vergleich mit inneren Idealen innerhalb des Störungsmodells eine wichtige Funktion. Die Internalisierung medialer Idealbilder könnte innere Ideale maßgeblich prägen und diese stabilisieren.

Die Wirkrichtung der Zusammenhänge bleibt in den bisherigen Untersuchungen unklar. Eine bidirektionale Interaktion ist aus theoretischer Sicht wahrscheinlich. Einerseits kann die Instagram-Exposition unter bestimmten Bedingungen zu einer Verstärkung der Symptomatik führen. Andererseits suchen Personen mit hohem Streben nach Muskulosität möglicherweise entsprechende Inhalte häufiger auf. Daraus lässt sich eine aufrechterhaltende Funktion des Nutzens sozialer Medien für die muskeldysmorphe Symptomatik ableiten. Um die Wirkzusammenhänge besser zu verstehen, sollten die Hypothesen auch mithilfe experimenteller Methoden untersucht werden.

\section{Fazit für die Praxis}

- Die Studie bietet erste Hinweise, dass Personen, die ausschließlich Kraftsport ausüben, ein höheres Risiko für eine muskeldysmorphe Symptomatik mitbringen könnten. Ballsportler, die ebenfalls Kraftsport ausüben, scheinen hingegen weniger risikobehaftet zu sein.

- Sowohl die allgemeine als auch die spezifische Nutzung von Instagram könnte zur Aufrechterhaltung der Problematik beitragen und sollte aus diesem Grund im Rahmen der Therapie in ihrer Funktionalität angesprochen werden.

- Die Tendenz zu aussehensbezogenen Vergleichen spielt bei Männern bei der Betrachtung von spezifischen Fitspiration-Bildern eine Rolle.
- Die Internalisierung des medialen muskulösen Schönheitsideals ist ein wichtiger mediierender Faktor, an dem im Rahmen der Therapie angesetzt werden sollte.

\section{Korrespondenzadresse}

Dr. Katrin Schoenenberg

Klinische Psychologie und Psychotherapie, Bergische Universität Wuppertal

Max-Horkheimer-Str. 20, 42097 Wuppertal, Deutschland

schoenenberg@uni-wuppertal.de

Danksagung. Für ihre Unterstützung bei der Versuchsdurchführung bedanken wir uns bei Theresa Tabbica.

Funding. Open Access funding provided by Projekt DEAL.

\section{Einhaltung ethischer Richtlinien}

Interessenkonflikt. K. Schoenenberg und A. Martin geben an, dass kein Interessenkonflikt besteht.

Alle beschriebenen Untersuchungen am Menschen oder an menschlichem Gewebe wurden mit Zustimmung der zuständigen Ethikkommission, im Einklang mit nationalem Recht sowie gemäß der Deklaration von Helsinki von 1975 (in der aktuellen, überarbeiteten Fassung) durchgeführt. Von allen beteiligten Patienten liegt eine Einverständniserklärung vor.

Open Access. Dieser Artikel wird unter der Creative Commons Namensnennung 4.0 International Lizenz veröffentlicht, welche die Nutzung, Vervielfältigung, Bearbeitung, Verbreitung und Wiedergabe in jeglichem Medium und Format erlaubt, sofern Sie den/die ursprünglichen Autor(en) und die Quelle ordnungsgemäß nennen, einen Link zur Creative Commons Lizenz beifügen und angeben, ob Änderungen vorgenommen wurden.

Die in diesem Artikel enthaltenen Bilder und sonstiges Drittmaterial unterliegen ebenfalls der genannten Creative Commons Lizenz, sofern sich aus der Abbildungslegende nichts anderes ergibt. Sofern das betreffende Material nicht unter der genannten Creative Commons Lizenz steht und die betreffende Handlung nicht nach gesetzlichen Vorschriften erlaubt ist, ist für die oben aufgeführten Weiterverwendungen des Materials die Einwilligung des jeweiligen Rechteinhabers einzuholen.

Weitere Details zur Lizenz entnehmen Sie bitte der Lizenzinformation auf http://creativecommons.org/ licenses/by/4.0/deed.de.

\section{Literatur}

American Psychiatric Association (2013) Diagnostic and statistical manual of mental disorders, 5. Aufl. American Psychiatric, Arlington

Van Den Berg P, Thompson JK, Obremski-Brandon K, Coovert M (2002) The tripartite influence model 
of body image and eating disturbance: a covariance structure modeling investigation testing the mediational role of appearance comparison. J Psychosom Res 53(5):1007-1020

Blond A (2008) Impacts of exposure to images of ideal bodies on male body dissatisfaction: a review. Body Image 5(3):244-250

Bortz J, Schuster C (2010) Tests zur Überprüfung von Unterschiedshypothesen. In: Bortz J, Schuster C (Hrsg) Statistik für Human- und Sozialwissenschaftler. Springer, Berlin, Heidelberg, New York, S117-136

Brown Z, Tiggemann M (2016) Attractive celebrity and peer images on Instagram: effect on women's mood and body image. Body Image 19:37-43

Brown TA, Forney KJ, Pinner D, Keel PK (2017) A randomized controlled trial of the body project: more than muscles for men with body dissatisfaction. Int JEat Disord 50(8):873-883

Cohen R, Newton-John T, Slater A (2017) The relationship between Facebook and Instagram appearance-focused activities and body image concerns in young women. Body Image 23:183-187

Fardouly J, Willburger BK, Vartanian LR (2018) Instagram use and young women's body image concerns and self-objectification: Testing mediational pathways. New Media Soc 20(4):1380-1395

Festinger $L$ (1954) A theory of social comparison processes. Hum Relat 7(2):117-140

FrederickDA, Buchanan GM, Sadehgi-AzarL,PeplauLA, Haselton MG, Berezovskaya A, Lipinski RE (2007) Desiring the muscular ideal: Men's body satisfaction in the United States, Ukraine, and Ghana. Psychol Men Masc 8(2):103-117

Groesz LM, Levine MP, Murnen SK (2002) The effect of experimental presentation of thin media images on body satisfaction: a meta-analytic review. Int J Eat Disord 31(1):1-16

Hausenblas HA, Campbell A, Menzel JE, Doughty J, Levine M, Thompson JK (2013) Media effects of experimental presentation of the ideal physique on eating disorder symptoms: a metaanalysis of laboratory studies. Clin Psychol Rev 33(1):168-181

Hayes AF (2019) The PROCESS macro for SPSS and SAS. http://processmacro.org/index.html. Zugegriffen:03.12.2018

Hildebrandt T, Langenbucher J, Schlundt DG (2004) Muscularity concerns among men: development of attitudinal and perceptual measures. Body Image 1(2):169-181

Holland G, Tiggemann M (2016) A systematic review of the impact of the use of social networking sites on body image and disordered eating outcomes. Body Image 17:100-110

Junco R, Heiberger G, Loken E (2011) The effect of Twitter on college student engagement and grades. JComput Assist Learn 27(2):119-132

Karazsia BT, Crowther JH (2009) Social body comparison and internalization: mediators of social influences on men's muscularity-oriented body dissatisfaction. Body Image 6(2):105-112

Klimek P, Murray SB, Brown T, Gonzales IVM Blashill AJ (2018) Thinness and muscularity internalization: associations with disordered eating and muscle dysmorphia in men. Int J Eat Disord 51(4):352-357

Knauss C, Paxton SJ, Alsaker FD (2009) Validation of the German version of the Sociocultural Attitudes Towards Appearance Questionnaire (SATAQ-G). Body Image 6(2):113-120
López-Guimerà G, Levine MP, Sánchez-Carracedo D, Fauquet J (2010) Influence of mass media on body image and eating disordered attitudes and behaviors in females: a review of effects and processes. Media Psychol 13(4):387-416

McCreary DR (2007) The drive for muscularity scale: description, psychometrics, and research findings. In: Thompson J, Cafri G (Hrsg) The muscular ideal: psychological, social, and medical perspectives. American Psychological Association, Washington, S87-106

McCreary DR, Sasse DK (2000) An exploration of the drive formuscularity in adolescent boys and girls. J Am Coll Health 48:297-304

Mölbert SC, Hautzinger M, Karnath HO, Zipfel S, Giel K (2017) Validation of the Physical Appearance Comparison Scale (PACS) in a German sample: psychometric properties and association with eating behavior, body image and self-esteem. Psychother Psychosom Med Psychol 67(2):91-97

Murray SB, Rieger E, Hildebrandt T, Karlov L, Russell J, Boon E, Dawson RT, Touyz SW (2012) A comparison of eating, exercise, shape, and weight related symptomatology in males with muscle dysmorphia and anorexia nervosa. Body Image 9(2):193-200

Myers TA, Crowther JH (2009) Social comparison as a predictor of body dissatisfaction: a meta-analytic review. J Abnorm Psychol 118(4):683-698

Olivardia R, Pope HG Jr, Borowiecki JJ 3rd, Cohane GH (2004) Biceps and body image: the relationship between muscularity and self-esteem, depression, and eating disorder symptoms. Psychol Men Masc 5(2):112-120

Pope HG Jr, Gruber AJ, Mangweth B, Bureau B, Decol C, Jouvent R, Hudson JI (2000) Body image perception among men in three countries. Am J Psychiatry 157(8):1297-1301

Schieber K, Kollei I, de Zwaan M, Martin A (2015) Classification of body dysmorphic disorder-What is the advantage of the new DSM-5 criteria? JPsychosom Res 78(3):223-227

Thompson JK, Cafri G (2007) The muscular ideal: psychological, social and medical perspectives. American Psychological Association, Washington

Tiggemann M, Miller J (2010) The Internet and adolescent girls' weight satisfaction and drive for thinness. Sex Roles 63(1-2):79-90

Tylka TL (2011) Refinement of the tripartite influence model for men: dual body image pathways to body change behaviors. Body Image 8(3):199-207

Waldorf M, Cordes M, Vocks S, McCreary D (2014) „Ich wünschte, ich wäre muskulöser": Eine teststatistische Überprüfung der deutschsprachigen Fassung der Drive for Muscularity Scale (DMS). Diagnostica 60(3):140-152

Weineck J (2010) Optimales Training, 16. Aufl. Spitta, Balingen

\section{Doktor Google}

Internetrecherche nach Krankheitssymptome wirkt sich negativ auf Psyche aus

Bereits eine kurze Internetsuche nach den empfundenen Symptomen kann die eigene Sorge, ernsthaft erkrankt zu sein, direkt steigern. Den Effekt von »Doktor Google« weist eine aktuelle Studie aus der Arbeitsgruppe um Professor Dr. Alexander Gerlach vom Lehrstuhl für Klinische Psychologie und Psychotherapie der Universität zu Köln nach.

Die Wissenschaftler ließen vorrangig junge Erwachsene im Alter von durchschnittlich 23 Jahren fünf Minuten lang persönliche Symptome im Internet suchen. Obwohl in dieser Altersgruppe das Risiko für eine Krankheitsangststörung üblicherweise gering ist, gaben die Probanden direkt nach der Suche an, sich nun mehr Sorgen über ihre Gesundheit und die Krankheitssymptome zu machen. Dabei führte das Googeln zu größerer Besorgnis, wenn die Teilnehmer bereits zuvor über eine negative Stimmung berichtet hatten. Die nachteiligen Folgen der Internetrecherche zeigten sich nicht nur, wenn Studienteilnehmer auf Internetseiten landeten, die über besonders gravierende Krankheiten informierten. Auch bei Webseiten mit zurückhaltenden, moderaten Auskünften zu Symptomen steigerte sich das Unwohlsein der Probanden.

Ganze 46 Prozent der Deutschen geben an, regelmäßig im Internet Gesundheitsthemen zu recherchieren. Da Langzeitstudien weitgehend fehlen, können zurzeit zwar keine Aussagen über einen Beitrag von Internetrecherchen zur Entwicklung einer Krankheitsangststörung (ehemals "Hypochondrie«) gemacht werden. Dass Recherchen sich negativ auf das psychische Wohlbefinden auswirken, konnte die Studie allerdings belegen.

Zur Publikation: Pollklas, M., Widemann, L. Lochschmidt, M., Plakhuta, A., \& Gerlach, A. L. (in press). Cyberchondriasis The Effect of Searching the Internet on Health Concerns. Zeitschrift für Psychologie [https://www.hf.uni-koeln.de/file/10825]

Quelle: Universität zu Köln, 19.02.2020 (https://portal.unikoeln.de) 